\title{
The nature and role of mucosal damage in relation to Salmonella typhimurium-induced fluid secretion in the rabbit ileum
}

\author{
T. S. WALLIS, W. G. STARKEY, J. STEPHEN, S. J. HADDON, ${ }^{*}$ M. P. OSBORNE* and D. C. A. \\ CANDY†
}

Departments of Microbiology and *Physiology, University of Birmingham, P.O. Box 363, Birmingham B15 2TT and + Institute of Child Health, Francis Rd, Birmingham B16 $8 E T$

Summary. The time course and nature of mucosal damage induced in rabbit ileal loops
by two strains of Salmonella typhimurium (TML and W118) isolated from human
infections was assessed by immunofluorescence microscopy and by scanning and
transmission electronmicroscopy. Salmonella-induced fluid secretion occurred in the
presence or absence of gross mucosal architectural damage. Neither strain caused
mucosal ulceration. When damage did occur, the villi were shortened by loss of their
tip regions with concomitant reforming of an intact mucosal surface. Immediately
preceding the onset of fluid secretion, marked infiltration of the mucosa with
polymorphonuclear leukocytes and occasional macrophages was seen. This revives an
earlier suggestion that interaction between invading salmonellae and acute inflamma-
tory cells may be an important factor in initiation of fluid secretion. Brush-border
invasion by salmonellae cannot per se be the immediate cause of fluid secretion,
because the latter occurred several hours after initial invasion.

\section{Introduction}

Salmonella-induced diarrhoea is a complex phenomenon in which several mechanisms may play a part (Stephen et al., 1985). In a previous paper (Wallis et al., 1985) we described the existence of salmonella enterotoxin(s), the significance of which in the causation of fluid secretion in experimental animals is now being investigated. In this paper we describe experiments designed to define the nature of, and evaluate the possible role of mucosal damage in, Salmonella-induced fluid secretion.

Various animal models have been used to study fluid secretion, or histological changes, or both, induced by salmonellae-rabbits (Taylor and Wilkins, 1961; Giannella et al., 1973), opiate-treated, starved guinea pigs (Takeuchi, 1967), rats (Powell et al., 1971), Rhesus monkeys (Rout et al., 1974) and chicks (Popiel and Turnbull, 1985). In these studies no systematic attempt was made to correlate the severity and nature of tissue damage with the onset of fluid secretion. Giannella and his coworkers did report qualitative differences in rabbit ileal-tissue damage induced by strains differing in virulence for

Received 18 Sep. 1985; accepted 10 Oct. 1985

Correspondence should be sent to Dr J. Stephen, Department of Microbiology, University of Birmingham, Birmingham B15 2TT. monkeys (Rout et al., 1974) and rabbits (Giannella et al., 1973). Based on limited histological observations they suggested that fluid secretion in the rabbit model preceded the onset of structural damage to the mucosa. No kinetic data were reported for monkeys (Rout et al., 1974); observations were made only at the peak of clinical diarrhoea.

In this paper we describe the time course of fluid secretion in rabbit ileal loops (RILs) infected with virulent $S$. typhimurium (strains TML and W118; Giannella et al., 1973) and relate this to mucosal damage as demonstrated by light and immunofluorescence microscopy and by scanning and transmission electronmicroscopy.

\section{Materials and methods}

\section{Bacterial strains}

$S$. typhimurium strains TML and W118, generously provided by Drs R. A. Giannella and J. W. Peterson, had previously been shown to invade and to induce fluid secretion in RIL tests (RILTs); this biotypical behaviour has been confirmed (Wallis et al., 1985). Strains were stored at $-70^{\circ} \mathrm{C}$ in $1-\mathrm{ml}$ volumes of equal proportions of glycerol and Mueller-Hinton broth.

\section{Rabbits}

Dutch rabbits $(1.5-2.0 \mathrm{~kg})$ from Hyline Rabbits, 
Cheshire, were used for experiment 1 and New Zealand rabbits $(2.5-3.0 \mathrm{~kg})$ from Regal Rabbits, Surrey, were used for experiments 2 and 3 .

\section{Fluid secretion}

S. typhimurium strains were tested for the ability to induce fluid secretion in RILTs as described by Wallis et al. (1985). Cholera toxin (CT; Sigma) $1 \cdot 5 \mu \mathrm{g}$ was used as a positive control. Fluid secretion was measured as volume of fluid accumulated $(\mathrm{v}) /$ length of loop $(\mathrm{L})(\mathrm{ml} / \mathrm{cm})$.

\section{Invasiveness}

Strains of S. typhimurium were assessed for invasiveness by immunofluorescence microscopy and by scanning and transmission electronmicroscopy.

Immunofluorescence microscopy. S. typhimurium antigen and antiserum to $S$. typhimurium were prepared as described by Roschka (1950). Samples for immunofluorescence microscopy were prepared as follows. Rabbits were anaesthetised at intervals after loop infection. Ileal loops were excised, and three full-thickness biopsies ( $c$. $1 \mathrm{~cm}^{2}$ ) were removed from the loops and pinned flat on dental wax. Biopsies for examination by immunofluorescence microscopy were fixed in $95 \%$ ethanol overnight at $4 \mathrm{C}$. They were then embedded and sectioned in paraffin wax $(2-\mu \mathrm{m}$ sections). Sections for immunofluorescence microscopy were incubated at $56 \mathrm{C}$ for $1 \mathrm{~h}$, dewaxed and soaked in phosphate-buffered saline (PBS; $\mathrm{NaCl} 8.5 \mathrm{~g} / \mathrm{L}$, $\mathrm{Na}_{2} \mathrm{HPO}_{4} 1.07 \mathrm{~g} / \mathrm{L}, \mathrm{NaH}_{2} \mathrm{PO}_{4} \cdot 2 \mathrm{H}_{2} \mathrm{O} 0.39 \mathrm{~g} / \mathrm{L}$ in distilled water, $p \mathrm{H} \mathrm{7.1)}$ for $20 \mathrm{~min}$. The slides were then placed in a moist chamber and exposed to $0.05 \mathrm{ml}$ of guinea pig antiSalmonella antiserum (1 in 20 dilution) for $30 \mathrm{~min}$. The slides were then washed in PBS for $10 \mathrm{~min}$ and treated with sheep anti-guinea pig-FITC conjugate (Wellcome Diagnostics; 1 in 20 dilution in PBS) containing rhodamine (Difco; 1 in 20 dilution) for $30 \mathrm{~min}$ in a moist chamber. The sections were finally washed in PBS for 10 min, mounted under a coverslip with alkaline-buffered glycerol $\left(\mathrm{NaHCO}_{3} 0 \cdot 175 \mathrm{~g} / \mathrm{L}, \mathrm{Na}_{2} \mathrm{CO}_{3} 0 \cdot 16 \mathrm{~g} / \mathrm{L}\right.$, glycerol $90 \% \mathrm{v} / \mathrm{v}$ in distilled water) and examined immediately by fluorescence microscopy with a Zeiss UV microscope (450-490 $\mathrm{nm}$ barrier filters). The presence of bright greenfluorescing, rod-shaped bacteria in the mucosal cells and lamina propria was taken as evidence of mucosal invasion.

Scanning electronmicroscopy (SEM). Biopsies were washed thoroughly with Hartley digest broth before fixation to remove debris that might obscure surface detail of the villi. Biopsies were immersed for $1 \mathrm{~h}$ in glutaraldehyde $2.5 \%$ then post-fixed for $1 \mathrm{~h}$ in $\mathrm{OsO}_{4} 1 \%$. Glutaraldehyde and $\mathrm{OsO}_{4}$ were each made up in $0.05 \mathrm{M}$ phosphate buffer made up to a total molarity of $0.35 \mathrm{M}$ with sucrose. Biopsies were then immersed for $\mathrm{I} \mathrm{h}$ in thiocarbohydrazide $1 \%$ at $50 \mathrm{C}$, washed for $15 \mathrm{~min}$ in water at $50 \mathrm{C}$. osmicated for $1 \mathrm{~h}$ in $\mathrm{OsO}_{4} 2 \%$ in water at $50 \mathrm{C}$ (Ono and Takashio, 1978) and then dehydrated serially in acetone $25 \%, 50 \%$ and $100 \%$ before critical point drying with liquid $\mathrm{CO}_{2}$. The specimens were fixed to copper plates with electrically conducting paint (RS 555-
156) and examined without coating in a JEOL 120CX II electronmicroscope fitted with a TEMSCAN attachment. Images were obtained via a secondary electron detector at accelerating voltages of 20 or $40 \mathrm{kV}$.

Transmission electronmicroscopy (TEM). Biopsies were fixed as for SEM. After fixation they were dehydrated in ethanol and $1 \mathrm{~mm}^{3}$ portions were embedded in an epon-araldite mixture (Mollenhauer, 1964). Sections were cut and stained in methanolic uranyl acetate and lead citrate, and examined in a JEOL $120 \mathrm{CX}$ II electronmicroscope at accelerating voltages of 60 or $80 \mathrm{kV}$.

\section{Results}

\section{Time course of fluid secretion}

The time courses for fluid secretion induced by $S$. typhimurium strains TML, W118 and by CT in RILs are shown in fig 1 . Fluid secretion induced by CT was established by $8 \mathrm{~h}(\mathrm{~V} / \mathrm{L}=0.5 \mathrm{ml} / \mathrm{cm})$ and continued until at least $16 \mathrm{~h}$ after inoculation. $S$. typhimurium strains TML (two experiments) and W118 each induced net fluid secretion between 6 and $8 \mathrm{~h}$ which increased until at least $14 \mathrm{~h}$ after inoculation.

\section{Immunofluorescence microscopy}

S. typhimurium strain TML (experiment 1). Immunofluorescence microscopy of samples taken $2 \mathrm{~h}$ after inoculation showed individual Salmonella organisms invading the ileal mucosa (fig. $2 a$ ); this was confirmed by TEM (fig. $2 b$ ). Most organisms were still adherent to the brush border or free in the lumen (not shown in this field). By $4 \mathrm{~h}$, microcolonies of bacteria could be seen within enterocytes. The degree of invasion thereafter increased with time, though within each ileal loop the degree of villus invasion was not uniform and sections of some villi were entirely free of organisms. By $8 \mathrm{~h}$, a massive influx of infiltrating polymorphonuclear leukocytes (PMNLs) was seen. By $12 \mathrm{~h}$, in villi with heavily invaded tips, tip enterocytes containing bacteria were rounding up and were being extruded (fig. 2c). This extrusion process, followed by resealing of the damaged mucosal layer, resulted in villus shortening. By $18 \mathrm{~h}$, organisms could be seen towards the bases of villi (fig. $2 d$ ).

S. typhimurium strains $T M L$ (experiment 2) and W118 (experiment 3). The patterns of bacterial invasion were similar to that seen in experiment 1 .

\section{Scanning electronmicroscopy}

In all three experiments the mucosal surfaces of uninfected control loops were covered with con- 


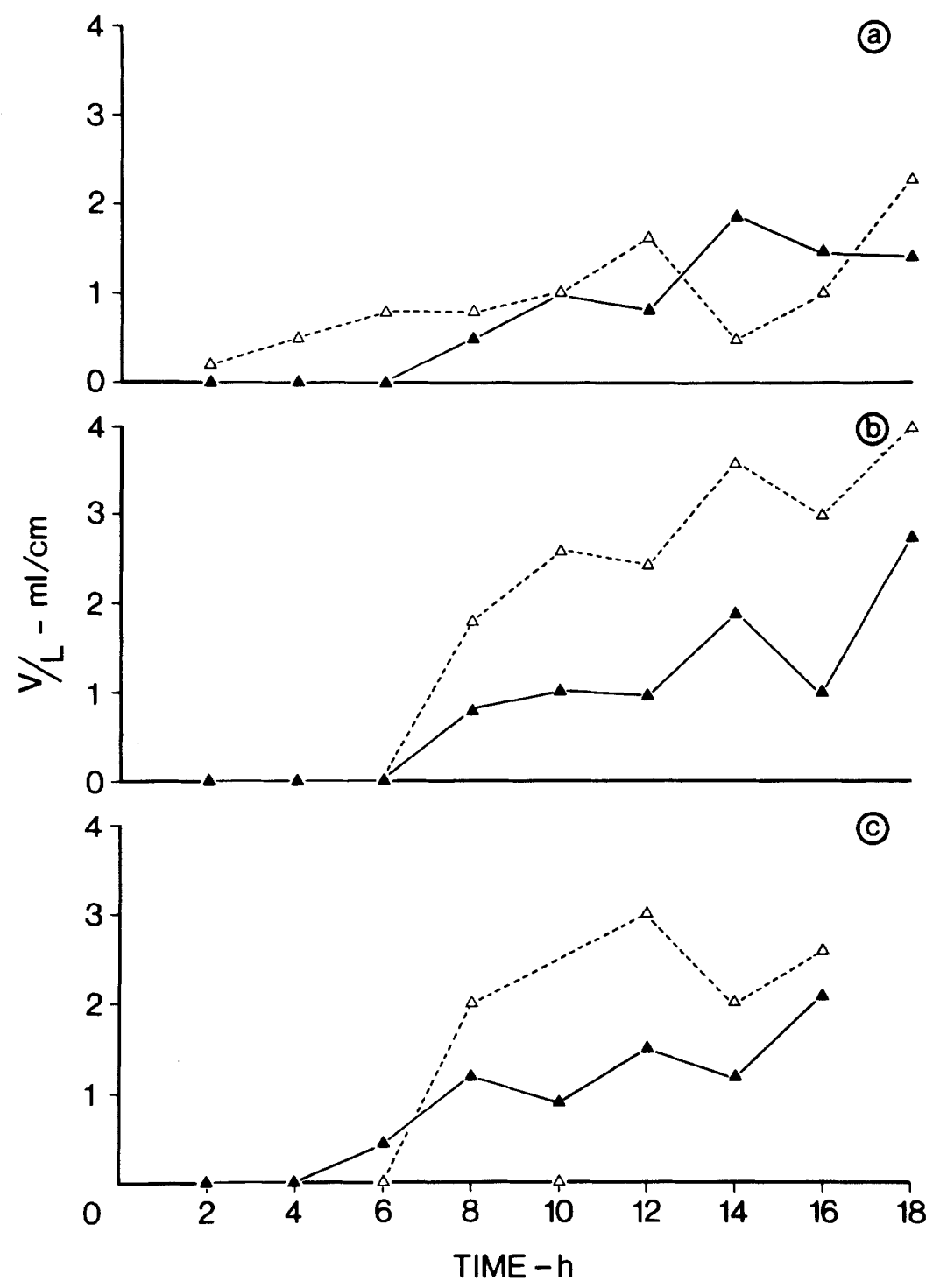

Fig. 1. Time courses of fluid secretion in rabbit ileal loops inoculated with $S$. typhimurium, strains TML (experiment 1 , fig. $1 a$; experiment 2, fig. $1 b$ ) and W118 (fig. $1 c$ ). Cholera toxin was used as a positive control. Data for each time point were obtained from individual rabbits, and are means derived from paired loops. $\Delta--\Delta$ Cholera toxin; $\Delta-\Delta S$ typhimurium.

siderably more mucus and entrapped material than were time-matched infected secreting loops from the same animals.

S. typhimurium strain TML (experiment 1). Little evidence of gross change in villus architecture was seen in loops infected with strain TML by $4-6 \mathrm{~h}$ (fig. $3 a$ ). By $8 \mathrm{~h}$, increased surface irregularity was apparent (fig. $3 b$ ). At $12 \mathrm{~h}$, extrusion of villus-tip material was obvious (fig. $3 c$ ). Mucosal damage was maximal at $16 \mathrm{~h}$; villi were shortened and blunted and there was extensive shedding of villus-tip material (fig. 3d). There was evidence of recovery of villus surface structure at $18 \mathrm{~h}$ when compared with an 18-h negative control loop from the same animal (fig. $4 a, b$ ).

S. typhimurium strain $T M L$ (experiment 2). No obvious difference between infected and control mucosae was observed until $8 \mathrm{~h}$ when villi from infected loops appeared comparable with those in fig. $3 b$. Significant extrusion of villus-tip material was observed only at $18 \mathrm{~h}$, when extrusion was much less than is indicated by fig. $3 c$ in experiment 1 ; 

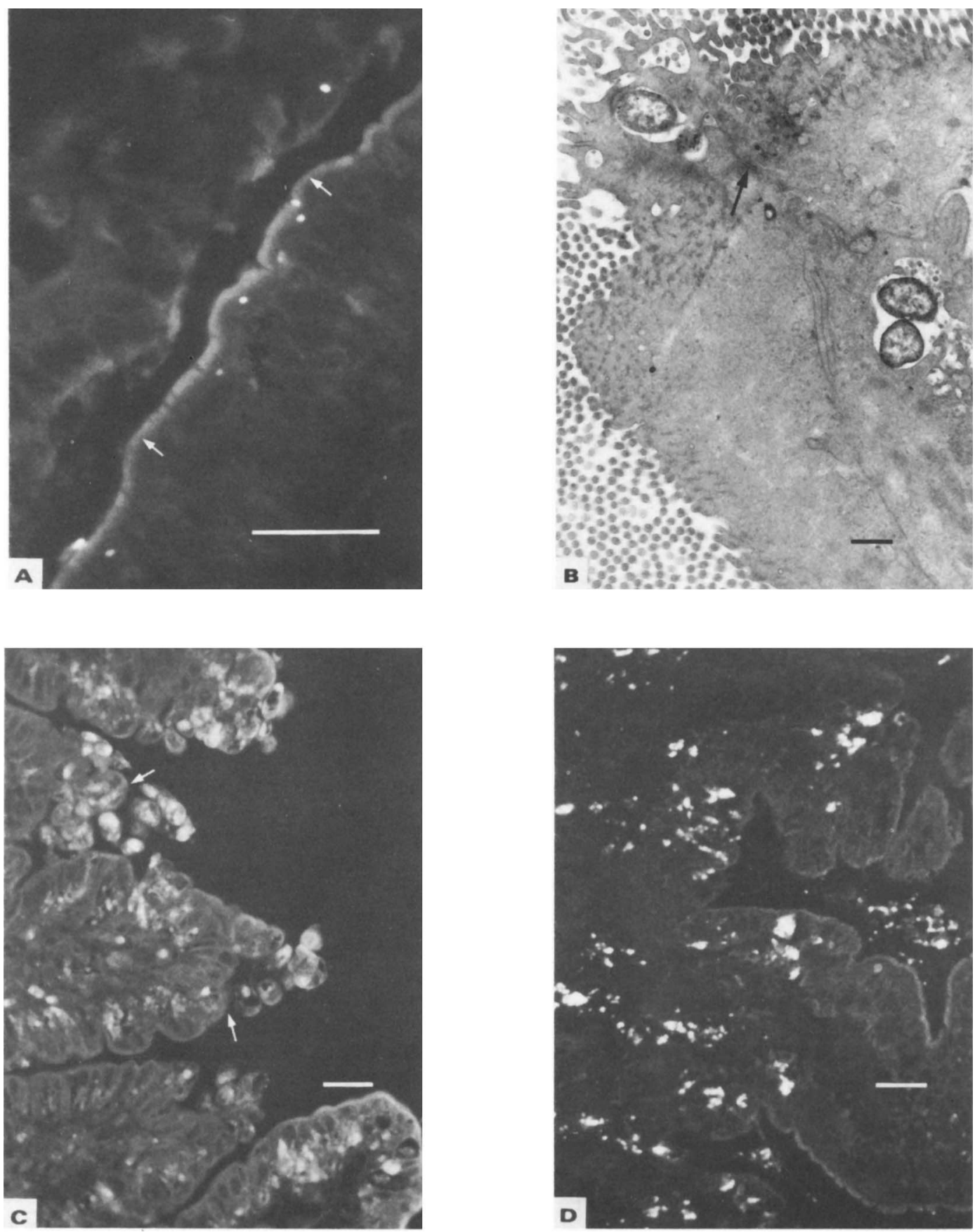

Fig. 2. Invasion of S. typhimurium. strain TML. experiment 1 (see fig. $1 a$ ). $a$. Individual organisms, visualised by immunofluorescence microscopy, invading the lateral region of a villus, $2 \mathrm{~h}$ after inoculation; $\rightarrow$ : brush border: bar $=10 \mu \mathrm{m}$. $b$. A similar preparation to that in $2 a$, taken $2 \mathrm{~h}$ after inoculation and examined by TEM. Note only local disturbance of brush border and invasion of enterocytes. Organisms were not seen penetrating through tight cell-cell junctions: $\rightarrow$ tight junction; bar $=1 \mu$ m. $c$. Fluorescent-antibody-stained section of $S$. typhimurium-infected loop, taken $12 \mathrm{~h}$ after inoculation. Note villus-tip enterocytes, laden with bacteria, rounded-up and being extruded. The mucosa appears to become rapidly resealed $(\rightarrow) ;$ bar $=1 \mu \mathrm{m}$. $d$. A similar preparation to that in $2 c$ taken at $18 \mathrm{~h}$. Note increased numbers of bacteria in the region of the bases of villi; bar $=10 \mu \mathrm{m}$. 

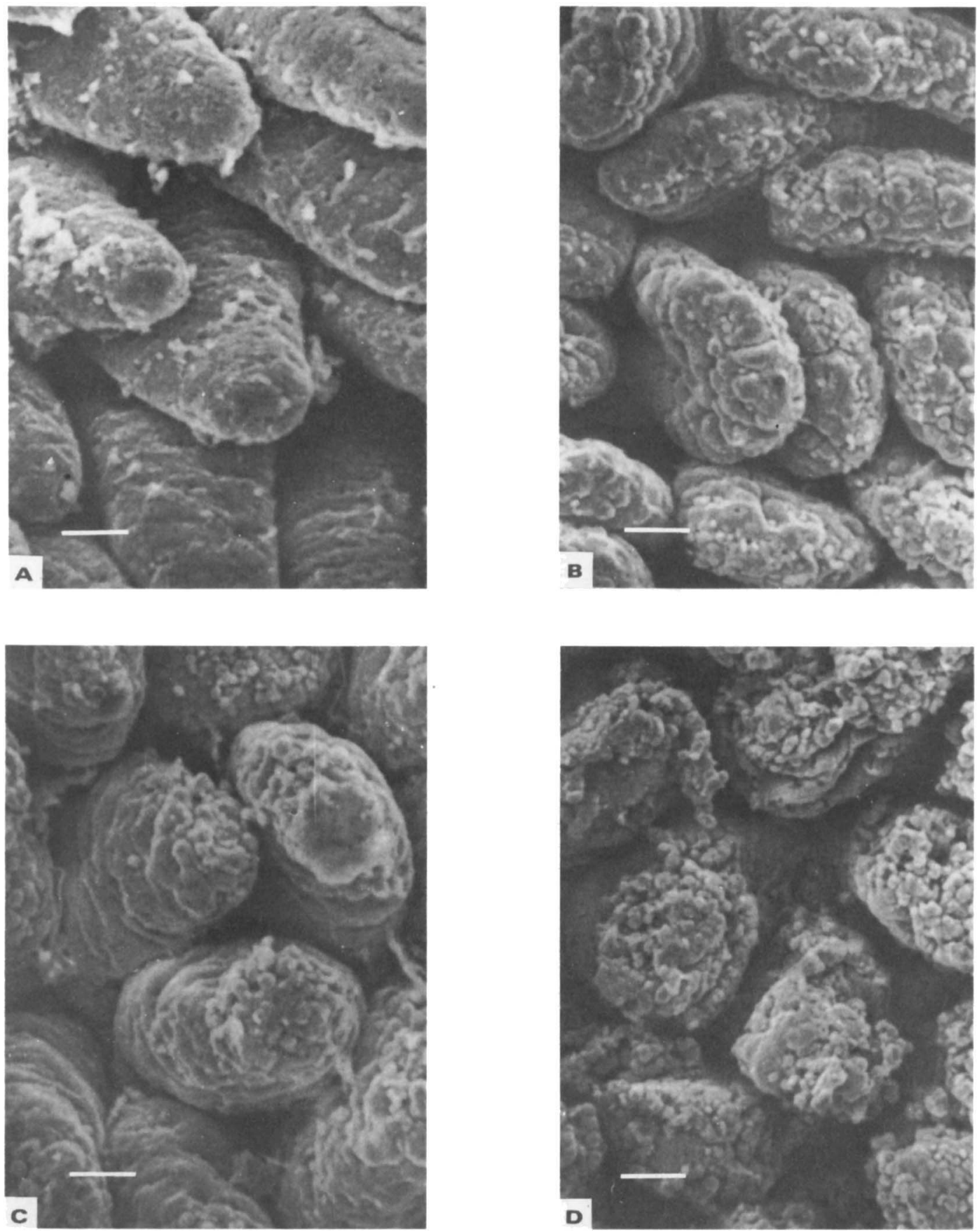

Fig. 3. SEM analysis of $S$. typhimurium-induced changes in villus architecture; experiment 1 (see fig. 1 a). Biopsies were taken from separate rabbits $4,8,12$ and $16 \mathrm{~h}$ after inoculation of RILs ( $a, b, c$ and $d$ respectively), processed and analysed by SEM. The appearances of villi ranged from normal $(a)$ through surface wrinkling $(b)$ to progressive shedding of tip cells $(c, d)$. Note the SEM appearance of $3 c$ and compare this with $2 c$. Fig. 3 (and also fig. 4) comprises representative regions of mucosa selected from a larger field at $400 \times$ magnification and containing around 50 villi. Bar $=100 \mu \mathrm{m}$. 

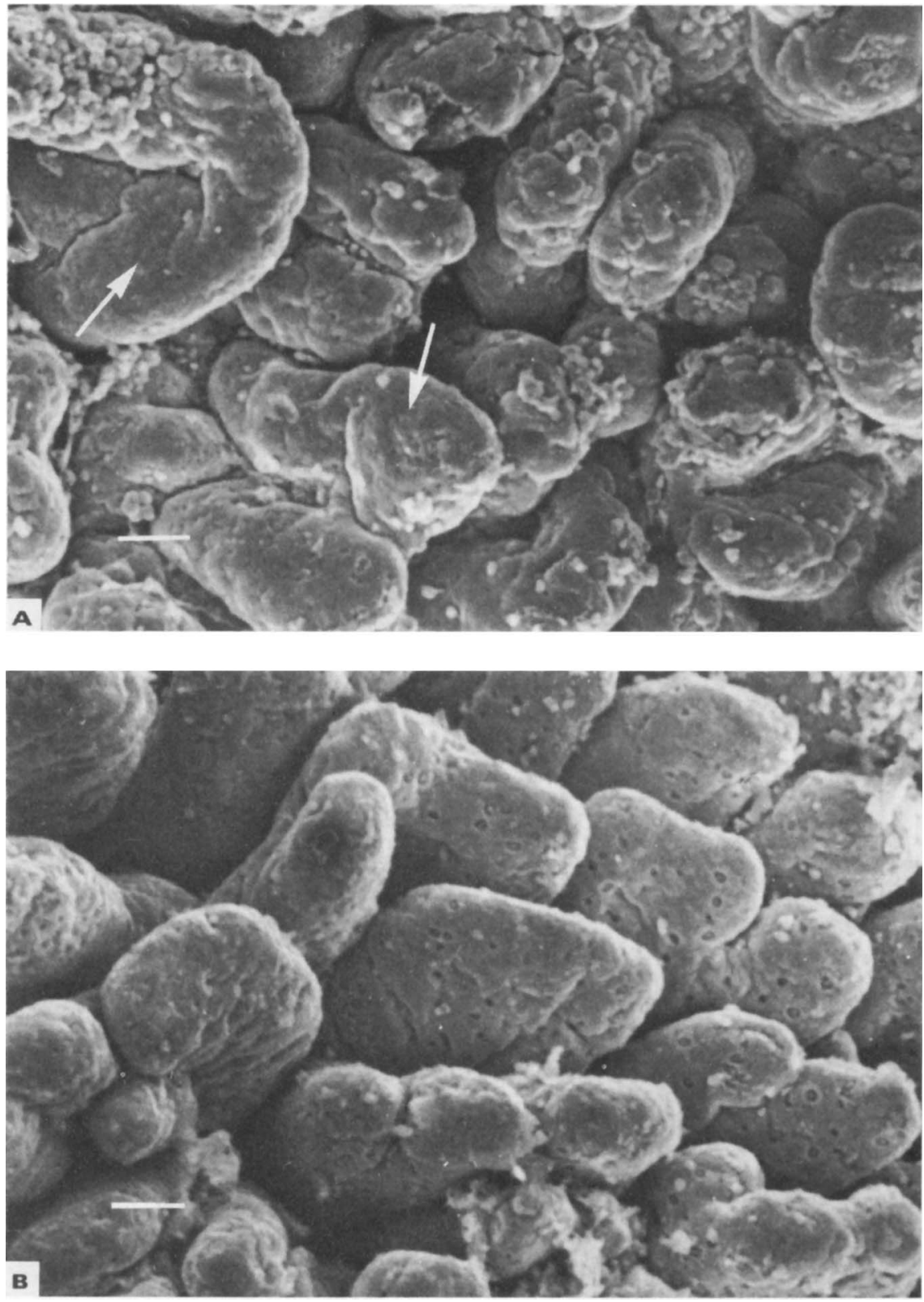

Fig. 4. SEM analysis of $S$. typhimurium-induced changes in villus architecture (experiment 1 ; see fig. la). Biopsies were taken from the same rabbits at $18 \mathrm{~h}:(a)$ after inoculation of RIL with $S$. typhimurium, and $(b)$ from a control loop that received sterile medium. Note: (i) evidence of recovery of villi, which, though shortened, show less tip derangement and a return to more normal surface appearances $(\rightarrow)$. (ii) Ligation for $18 \mathrm{~h}$ per se does not induce changes in villus surface structure. The picture shown was virtually indistinguishable from control biopsies taken at $0 \mathrm{~h}$. Fig. 4 (and also fig. 3) comprises representative regions of mucosa selected from a large field at $400 \times$ magnification and containing around 50 villi. Bar $=100 \mu \mathrm{m}$.

gross villus-tip destruction, as seen in experiment 1 (fig. $3 d$ ), was not observed. No clear indication of shortening of villi was observed.

S. typhimurium strain W118 (experiment 3 ). The maximal change observed was seen at $10 \mathrm{~h}$; this was not progressive, as in experiment 1 . Villi appeared to exhibit surface irregularity (similar to fig $3 b$ ) but subsequent mucosal samples from animals at different time points were not significantly different from corresponding time-matched controls. There was less evidence of significant villus shortening than in experiment 1 . 
Transmission electronmicroscopy

S. typhimurium strain TML (experiment 1). Invasion of ileal mucosa by strain TML was observed by $2 \mathrm{~h}$ after inoculation (fig. $2 b$ ), but with
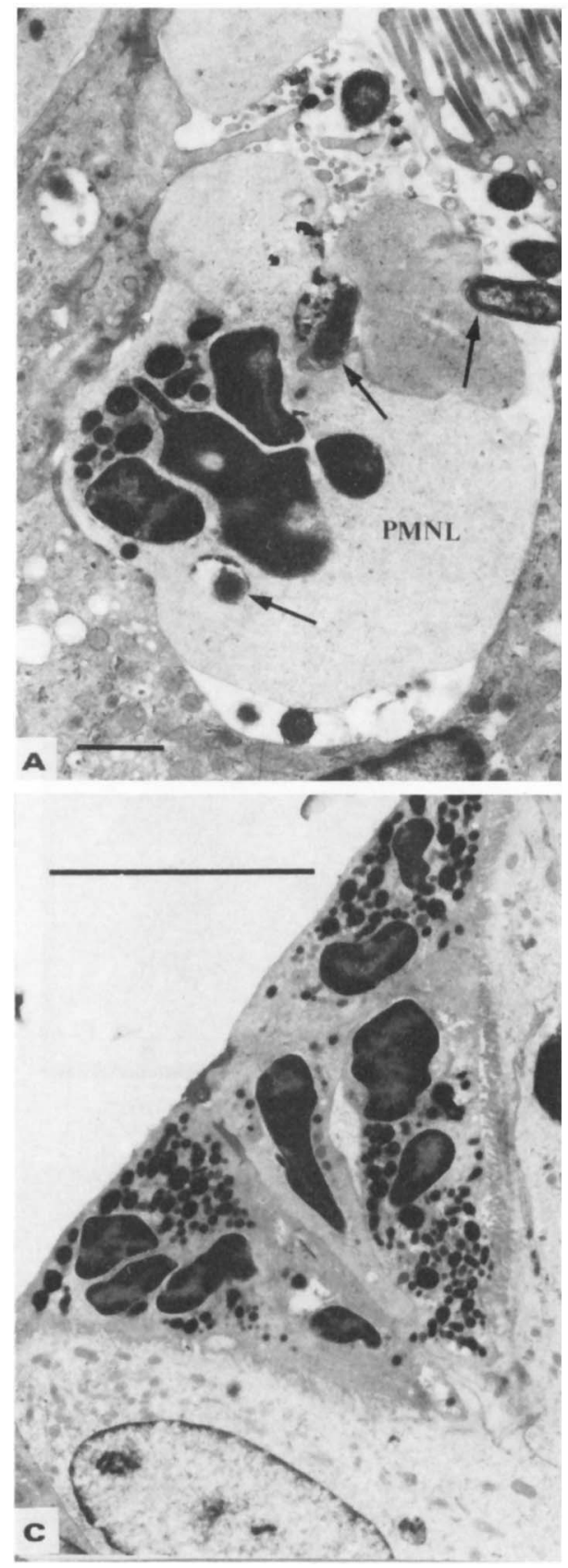

mainly local derangement of the brush border during invasion of ileal enterocytes. By $4 \mathrm{~h}$, microcolonies of TML were seen in intra-cytoplasmic vacuoles containing remnants of microvilli and also in the cytoplasm; these findings were similar to
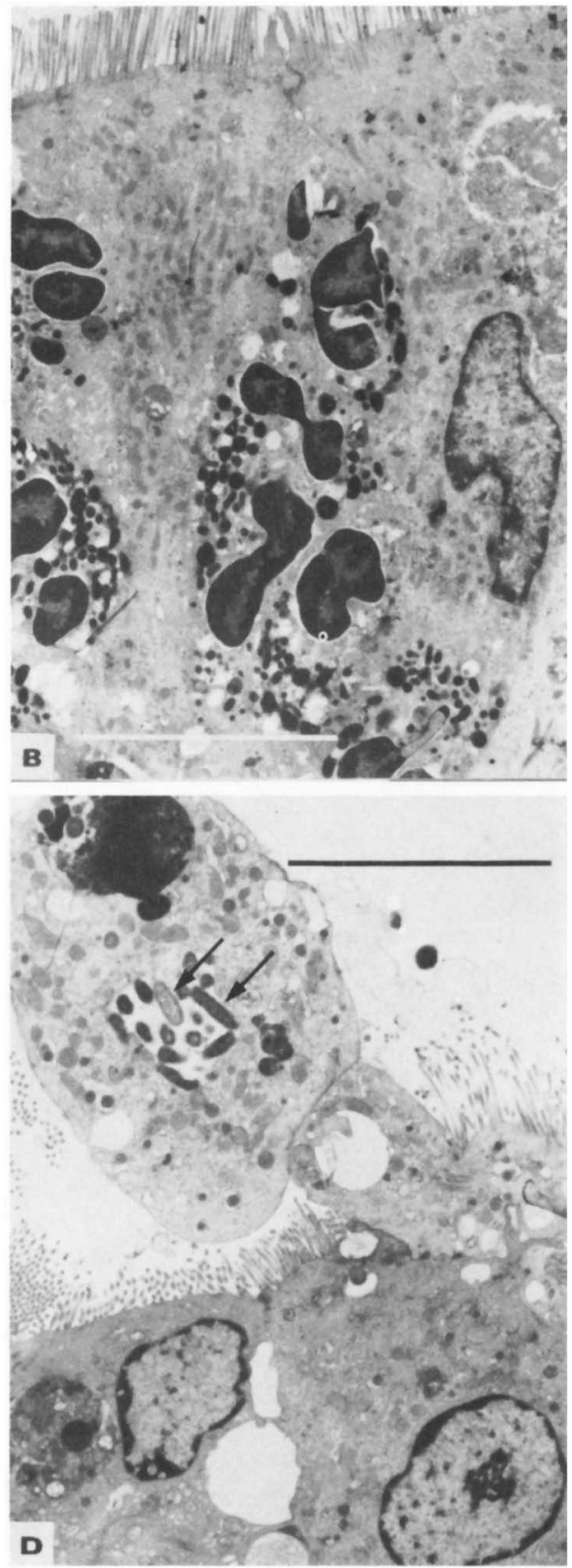

Fig. 5. TEM analysis of tissues infected with $S$. typhimurium (experiment 1 ; see fig. 1a). Biopsies were taken $6,8,8$, and 14 h ( $a, b, c$ and $d$ respectively) after inoculation of RILs, from three different rabbits. $a$. PMNL interacting with bacteria $(\rightarrow)$ at surface of enterocyte layer; bar $=1 \mu \mathrm{m} . b$. Massive infiltration of PMNLs into mucosa between adjacent enterocytes; bar $=10 \mu \mathrm{m} . c$. PMNLs apparently being extruded into the lumen; bar $=10 \mu \mathrm{m}$. $d$. Extrusion of rounded-up, bacteria-containing enterocyte $(\rightarrow)$ (to be compared with figs. $2 c$ and $3 c, d)$; bar $=10 \mu \mathrm{m}$. Note: the cell-cell junctions appear to remain intact until the final expulsion of the infected enterocyte. 


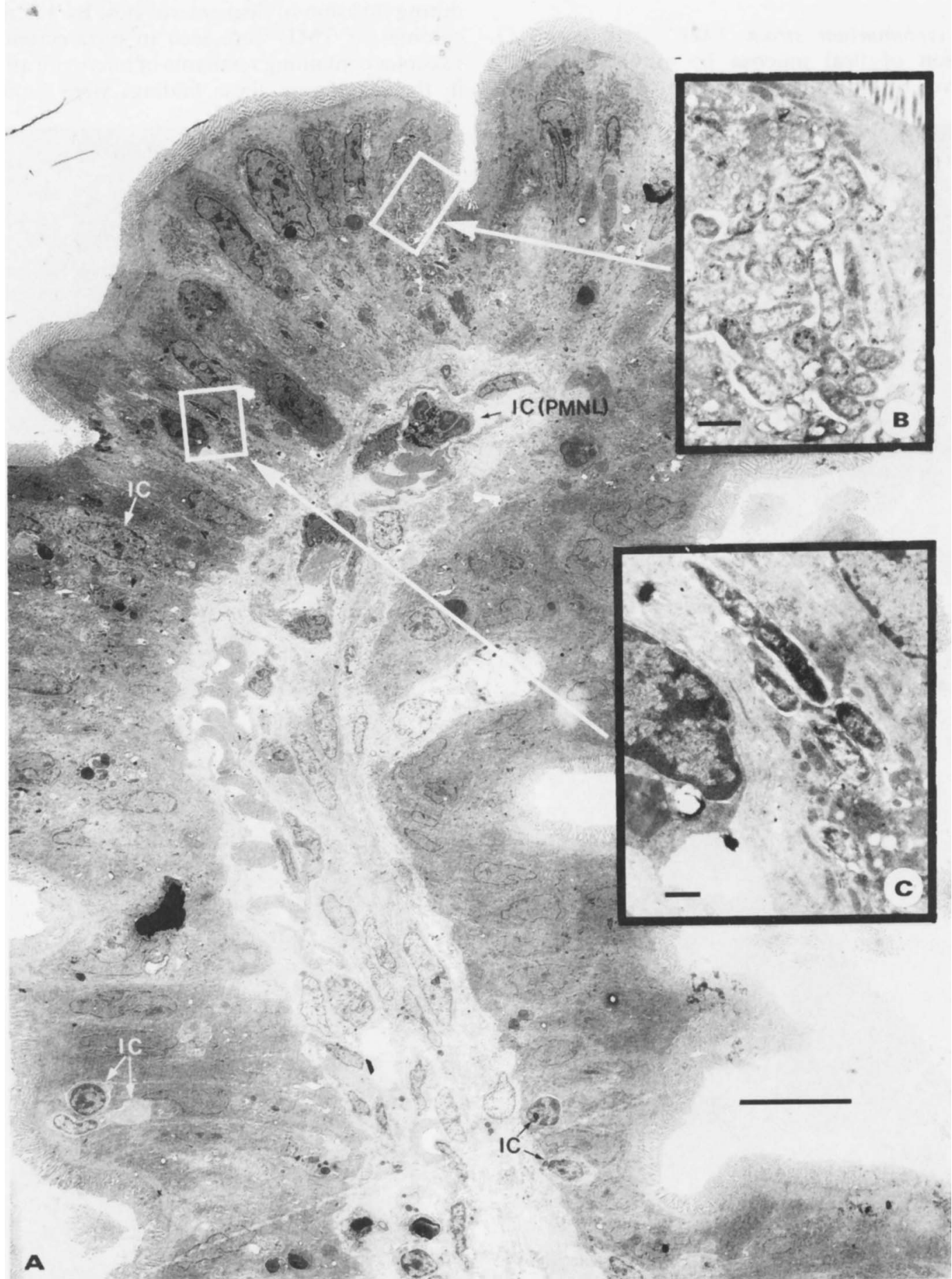

Fig. 6. TEM analysis of S. typhimurium-infected villus (experiment 2: fig $\mathrm{l} b$ ). Biopsy was taken $8 \mathrm{~h}$ after inoculation of a RIL. Note: (i) highly invaginated surface of enterocyte layer (a) (compare with fig. $3 b$ ); (ii) large numbers of invading $(b)$ and dividing $(c)$ bacteria; (iii) evidence for infiltrating cells of the immune system (IC $\rightarrow$ ): $\mathrm{bar}=10 \mu \mathrm{m}$. 
those recorded below for experiment 2 (figs. $6 a-c$ ). By $6 \mathrm{~h}$, phagocytosis of invading bacteria by PMNLs was observed (fig. $5 a$ ), and by $8 \mathrm{~h}$, large numbers of PMNLs were infiltrating the mucosa (fig. $5 b$ ) and extruding on to the mucosal surface (fig. $5 c$ ). At 12,14, 16 and $18 \mathrm{~h}$, many bacteria could be seen within severely damaged enterocytes with disrupted brush borders; some enterocytes were being extruded into the lumen from the tip region (fig. $5 d$ ).

S. typhimurium strain TML (experiment 2). The general patterns of bacterial invasion and PMNL infiltration already described for experiment 1 were again observed. Several features may be highlighted from micrographs of biopsies taken $8 \mathrm{~h}$ after inoculation. The highly invaginated outline of the longitudinal section (fig. $6 a$ ) corresponded with an SEM appearance similar to that in fig. $3 b$. Large numbers of intramucosal bacteria (figs. $6 b, c$ ) some of which were clearly dividing (fig. $6 c$ ) were observed; the latter were dividing in a cell with an intact brush border. It is not possible to be certain whether these organisms were being translocated into the lamina propria or were about to be expelled in rounded-up enterocytes. There was clear evidence in this and in many other sections of resident intra-epithelial lymphocytes and other infiltrating cells of the immune system.

S. typhimurium strain W118 (experiment 3). TEM appearances broadly resembled those seen with strain TML in experiment 2.

In all three experiments, sections through crypt regions revealed crypt cells with intact morphology without evidence of bacterial invasion.

\section{Discussion}

The onset of fluid secretion took place in all three experiments before (experiment 1), or in the absence of (experiment 2 and 3), the appearance of gross architectural damage to villi. The major difference between experiments 1 and 2, in which the same strain (TML) of S. typhimurium was used, was a different source and breed of rabbits. Thus, gross mucosal damage is not a prerequisite for Salmonella-induced fluid secretion and, when it occurs, may be host-dependent.

The methods used to analyse tissue damage can profoundly affect the perception of the nature and extent of morphological changes. For example, SEM, which in any one field gives an overview of around 50 villi at a magnification of $\times 400$, readily permits assessment of the proportion of damaged villi as judged by surface appearance (figs. 3 and 4). Importantly, the technique permits a more accurate evaluation of the frequency of damaged villus tips and hence the weight that should be given to the frequency of individual damaged enterocytes - seen in transmission electronmicrographs - in relation to pathophysiological changes. Indeed, without SEM, it would have been possible, depending on the plane of sectioning of biopsies taken from early time points in experiment 1 , to have misinterpreted the extent of architectural derangement observed by TEM of villi from infected loops.

The notion that gross structural damage is not an essential prerequisite for fluid secretion in salmonella infections is in keeping with the key findings of Rout et al. (1974) who showed that Salmonellainduced tissue damage depended on the site of infection. Jejunal secretion occurred in S. typhimurium-infected Rhesus monkeys without jejunal inflammation, histological damage or bacterial colonisation and invasion, albeit at one fixed time point during the diarrhoeal illness, although these processes did take place in the distal intestine. The data presented here also help to explain the earlier work of Fromm et al. (1974) with Ussing Chambers, which first led to the suggestion that Salmonellainduced diarrhoea is a secretory diarrhoea. The intestinal mucosa was taken from RILs $18 \mathrm{~h}$ after infection with strain TML. By this time the mucosa - had it been damaged as indicated by figs. $3 a-d$-would have been sufficiently re-formed and intact to allow successful stripping of mucosa for mounting in Ussing chambers and detection of intestinal secretion (see below).

The nature of the structural damage to villi is worthy of further comment. It may be seen (fig. $2 c$ ) that when villus-tip cells are shed there is an apparent re-sealing of the mucosa. At no time was there evidence of massive stripping of enterocytes which would lead to mucosal ulceration. This interpretation is strengthened by scanning electronmicrographs (figs. 3 and 4) in which it can be seen that, at the height of fluid secretion, there is an apparent return towards normal surface appearances. It is likely that much of the tissue debris and mucus seen in these micrographs would be flushed away by intestinal secretion and peristalsis in the unligated intestine. Koo et al. (1984) suggested that Salmonella-induced damage was due to the production of a Shiga-like cytotoxin (i.e., a toxin which inhibits protein synthesis). They posed the question, unanswered as yet, as to why the nature and extent of mucosal damage is greater in shigellosis than in salmonellosis. There are several reasons why we believe that such a cytotoxin need not be involved. It is possible to re-interpret their data (table 1 and fig. 5; Koo et al., 1984) to show that salmonella 
infection did not inhibit protein synthesis in enterocytes infected in vivo and analysed in vitro. These authors comment on, but do not explain, the fact that their infected tip cell-fractions contained more protein than comparable control preparations. The simplest explanation for this is that the protein concentration reflects the number of cells present in the fractions. At $18 \mathrm{~h}$ there would be a massive number of tissue PMNLs, which would not synthesize much protein. The decrease in enterocyte alkaline phosphatase activity reported by Koo et al. (1984) is more likely to be associated with loss of villus tip cells, a possibility they acknowledged (see also fig. 3, above), rather than being due to inhibition of protein synthesis, the explanation they preferred. Although the RIL is a good model for salmonella ileitis (Giannella et al., 1973) in that virulence in this model correlated with virulence in monkeys, and by extension man, it is not a good model for shigellosis (Gemski et al., 1972; Levine et al., 1973). Much more marked changes occurred in villus architecture in Shigella dysenteriae-infected loops (Levine et al., 1973) than we have observed in $S$. typhimurium-infected loops. It is, therefore, unwise to compare salmonella and shigella infections in this model. Any perturbation of the integrity of enterocytes in the villus tip may signal accelerated enterocyte expulsion. We have observed a similar phenomena in an unrelated systemrotavirus-infected villi in neonatal mice (unpublished observations).

The rounding-up and extrusion of villus-tip cells followed by resealing of the damaged villus tips described above, resulted in villus shortening. Villus-tip enterocytes are thought to be the site of solute and electrolyte absorption (Field, 1979) and crypt cells the site of secretion of electrolytes and water. Hence damage to villus tips during experimental $S$. typhimurium infection could result in decreased absorption, thereby unmasking physiological crypt secretion. This is clearly not the initiating factor in fluid secretion, but the fluid secretory response could thus be amplified or prolonged if, or when, this process of villus shortening occurs.

In all experiments there was a massive influx of inflammatory cells. PMNLs have previously been suggested as the source of endogenous prostaglandin which, when released as a result of bacterium-

\section{REFERENCES}

Field M 1979 Mechanisms of action of cholera and Escherichia coli enterotoxins. American Journal of Clinical Nutrition 32:189-196.
PMNL interaction (Gots et al., 1974; Giannella, 1979), act as mediators of fluid secretion. Our results support a possible role for PMNLs in the induction of fluid secretion in that the inflammatory response occurred in all three experiments and at times preceding the onset of overt fluid secretion. These earlier observations are being repeated, extended and quantified to assess the possible significance of the influx of inflammatory cells in the causation of fluid secretion.

Initial brush border damage or penetration cannot be the cause of fluid secretion because the latter begins several hours after the primary invasion of organisms. However, since as far as is known all virulent salmonellae so far described are invasive, it is clear that the process of invasion and tissue translocation is an important preliminary step towards the expression of the virulent phenotype. It is not possible to be sure whether the organisms shown in fig. 6 are in the process of penetrating deeper, or are about to be expelled. If the latter, the organisms that eventually reach the lamina propria (fig. 2) may be those that invade the basal regions of villus epithelium, because there was little evidence, even in experiment 1 , of expulsion of infected enterocytes from the basal regions. The somewhat more dramatic pictures reported by Takeuchi (1967) of "swelling and elongation of microvilli" coincident with the approach or adherence of Salmonella organisms were also seen in the present study but should not be regarded as typical, because the appearances seen in fig. $2 b$ were observed much more frequently.

Finally, the present study strongly suggests that damage of ileal villus architecture by $S$. typhimurium is confined to the tip regions and may be a nonspecific response to infection which expels invading pathogens. This damage is not essential for the induction of intestinal fluid secretion but the villus shortening which follows could give rise to a "physiological diarrhoea" which might be important in prolonging, though not initiating, fluid secretion.

JS, MPO and DCAC gratefully acknowledge the financial support of the Wellcome Trust. The authors also acknowledge the CIBA foundation for permission to publish figs. $5 b$ and $5 d$.

Fromm D, Giannella R A, Formal S B, Quijano R, Collins H 1974 Ion transport across isolated ileal mucosa invaded by salmonella. Gastroenterology 66:215-225.

Gemski P, Takeuchi A, Washington O, Formal S B 1972 Shigellosis due to Shigella dystenteriae 1: relative impor- 
tance of mucosal invasion versus toxin production in pathogenesis. Journal of Infectious Diseases 126:523-530.

Giannella R A 1979 Importance of the intestinal inflammatory reaction in salmonella-mediated intestinal secretion. Infection and Immunity 23:140-145.

Giannella R A, Formal S B, Dammin G J, Collins H 1973 Pathogenesis of salmonellosis. Studies of fluid secretion, mucosal invasion and morphologic reaction in the rabbit ileum. Journal of Clinical Investigation 52:441-453.

Gots R E, Formal S B, Giannella R A 1974 Indomethacin inhibition of Salmonella typhimurium, Shigella flexneri, and cholera-mediated rabbit ileal secretion. Journal of Infectious Diseases 130:280-284.

Koo F C W, Peterson J W, Houston C W, Molina N C 1984 Pathogenesis of experimental salmonellosis: inhibition of protein synthesis by cytotoxin. Infection and Immunity 43:93-100.

Levine M M et al. 1973 Pathogenesis of Shigella dysenteriae 1 (Shiga) dysentery. Journal of Infectious Diseases 127:261270.

Mollenhauer H H 1964 Plastic embedding mixtures for use in electron microscopy. Stain Technology 39:111-114.

Ono K, Takashio M 1978 Scanning electron microscopic studies of ileal epithelial cells in suckling rats. Anatomy and Embryology 153:1-8.

Popiel I, Turnbull P C B 1985 Passage of Salmonella enteritidis and Salmonella thompson through chick ileocecal mucosa. Infection and Immunity 47:786-792.
Powell D W, Plotkin G R, Maenza R M, Solberg L I, Catlin D H, Formal S B 1971 Experimental diarrhoea. I. Intestinal water and electrolyte transport in rat salmonella enterocolitis. Gastroenterology 60:1053-64.

Roschka R 1950 Eine neue Methode zur Herstellung Lochwertiger O-immunseren der Salmonella-grupe. Klinische Medizin (Wien) 5:88-93.

Rout W R, Formal S B, Dammin G J, Giannella R A 1974 Pathophysiology of salmonella diarrhoea in the rhesus monkey: intestinal transport, morphological and bacteriological studies. Gastroenterology 67:59-70.

Stephen J, Wallis T S, Starkey W G, Candy D C A, Osborne M P, Haddon S 1985 Salmonellosis: in retrospect and prospect. In: Bacterial toxins and diarrhoeal disease. Ciba Foundation Symposium 112, Pitman London, pp 175-193.

Takeuchi A 1967 Electron microscope studies of experimental Salmonella infection. I. Penetration into the intestinal epithelium by Salmonella typhimurium. American Journal of Pathology 50:109-136.

Taylor J, Wilkins M P 196I The effect of Salmonella and Shigella on ligated loops of rabbit gut. Indian Journal of Medical Research 49:544-549.

Wallis T S, Starkey W G, Stephen J, Haddon S J, Osborne M P, Candy D C A 1986 Enterotoxin production by strains of Salmonella typhimurium of different virulence. Journal of Medical Microbiology 21:19-23. 